\title{
Viscosity Solutions of Uniformly Elliptic Equations without Boundary and Growth Conditions at Infinity
}

\author{
G. Galise and A. Vitolo \\ Department of Mathematics, University of Salerno, 84084 Fisciano, Italy \\ Correspondence should be addressed to A. Vitolo, vitolo@unisa.it \\ Received 4 May 2011; Accepted 8 September 2011 \\ Academic Editor: Julio Rossi
}

Copyright (c) 2011 G. Galise and A. Vitolo. This is an open access article distributed under the Creative Commons Attribution License, which permits unrestricted use, distribution, and reproduction in any medium, provided the original work is properly cited.

We deal with fully nonlinear second-order equations assuming a superlinear growth in $u$ with the aim to generalize previous existence and uniqueness results of viscosity solutions in the whole space without conditions at infinity. We also consider the solvability of the Dirichlet problem in bounded and unbounded domains and show a blow-up result.

\section{Introduction and Statement of the Main Results}

We are concerned with the well-posedness of the fully nonlinear second-order uniformly elliptic problem

$$
F\left(x, u, D u, D^{2} u\right)=f(x) \text { in } \mathbb{R}^{n},
$$

with no limitation on the growth of $f$ and no condition on the behaviour of $u$ at infinity.

We will assume the following standard structure condition, which implies the uniform ellipticity:

$$
D_{\lambda, \Lambda}^{-}(Y-X)-\gamma|\eta-\xi| \leq F(x, u, \eta, Y)-F(x, u, \xi, X) \leq D_{\lambda, \Lambda}^{+}(Y-X)+\gamma|\eta-\xi|
$$

for $x \in \mathbb{R}^{n}$ and any $u \in \mathbb{R}, \xi, \eta \in \mathbb{R}^{n}, X, Y \in \mathcal{S}^{n}$. 
As regards the monotonicity in the variable $u$, we ask something more than the usual monotonicity assumption $(\delta=0)$ :

$$
F(x, u, \xi, X)-F(x, v, \xi, X) \leq-\delta(u-v)^{s} \quad \text { if } v<u
$$

for $s>1$ and $\delta>0$.

We also suppose, as generally with the formulation (1.1), that

$$
F(x, 0,0,0)=0
$$

We collect the above assumptions in the structure condition

$$
(\mathrm{SC}):=(1.2) \text { and (1.3) and (1.4), }
$$

noting that

$$
F\left(x, u, D u, D^{2} u\right)=D_{\lambda, \Lambda}^{+}\left(D^{2} u\right)+\gamma|D u|-|u|^{s-1} u
$$

fits into our framework.

Hence, this paper is in the wake of Brezis [1], who proved the existence and uniqueness of distributional solutions $u \in L_{\mathrm{loc}}^{s}\left(\mathbb{R}^{n}\right)$ for the semilinear equation

$$
\Delta u-|u|^{s-1} u=f(x) \quad \text { in } \mathbb{R}^{n}
$$

with $f \in L_{\text {loc }}^{1}\left(\mathbb{R}^{n}\right)$ and of Esteban et al. [2] for the case of $L^{n}$-viscosity solutions of the fully nonlinear second order uniformly elliptic equation

$$
F\left(D^{2} u\right)-|u|^{s-1} u=f(x)
$$

with $f \in L_{\text {loc }}^{n}\left(\mathbb{R}^{n}\right)$.

Our aim is to extend the result in different directions, including lower-order terms, allowing the dependence on $x$ and going below the exponent $n$.

Throughout the paper $\lambda$ and $\Lambda$ are positive constants such that $\Lambda \geq \lambda$, called ellipticity constants, and $\gamma \geq 0$ will play the role of a Lipschitz constant.

Also, $p_{0}=p_{0}(n, \Lambda / \lambda) \in(n / 2, n)$ is the exponent such that for $p>p_{0}$ the generalized maximum principle (GMP) holds true; see Escauriaza [3], Crandall and Swiech [4], and Koike and Swiech [5]: if $f \in L^{p}(\Omega)$ with $p>p_{0}$ and $u \in W_{\text {loc }}^{2, p}(\Omega) \cap C(\bar{\Omega})$ is an $L^{p}$-strong solution of the maximal equation

$$
p_{\lambda, \Lambda}^{+}\left(D^{2} u\right)+\gamma|D u| \geq f
$$


then

$$
\max _{\bar{\Omega}} u \leq \max _{\partial \Omega} u+C d^{2-(n / p)}\left\|f^{-}\right\|_{L^{p}(\Omega)}
$$

with $d=\operatorname{diam}(\Omega)$ and $C$ being a positive constant depending on $n, \lambda, \Lambda, p, \gamma d$.

Here and in the sequel by $L^{p}$-strong solutions of the equation $F\left(x, u, D u, D^{2} u\right)=f(x)$ we intend the $W_{\text {loc }}^{2, p}$ functions which are solutions almost everywhere with respect to the Lebesgue measure (almost everywhere) as well as classical solutions to be $C^{2}$ pointwise solutions.

We also call $L^{p}$-strong (classical) subsolutions, respectively supersolutions, of $F=f$ the $W_{\text {loc }}^{2, p}\left(C^{2}-\right)$ solutions of the inequalities $F\left(x, u, D u, D^{2} u\right) \geq f(x)$, respectively $F(x, u$, $\left.D u, D^{2} u\right) \leq f(x)$, almost everywhere (everywhere).

For the definition of $C$-viscosity and $L^{p}$-viscosity solutions, which are our main concern, we refer to Section 2. Correspondingly subsolutions, respectively supersolutions, in the viscosity sense will be referred to as solutions of the equation $F \geq f$, respectively $F \leq f$.

We establish a first result in the case of $F$ independent of $x$.

Theorem 1.1. Let $F: \mathbb{R}^{n} \times \mathbb{R} \times \mathbb{R}^{n} \times \mathcal{S}^{n} \rightarrow \mathbb{R}$ be a continuous function satisfying the structure condition (SC) such that

$$
F(x, u, \xi, X)=\bar{F}(u, \xi, X)
$$

for all $(x, u, \xi, X) \in \mathbb{R}^{n} \times \mathbb{R} \times \mathbb{R}^{n} \times \mathcal{S}^{n}$. If $f \in C\left(\mathbb{R}^{n}\right)$, there exists a unique $C$-viscosity solution of equation

$$
\bar{F}\left(u, D u, D^{2} u\right)=f(x) \text { in } \mathbb{R}^{n}
$$

To consider a dependence on $x$, we need to control the oscillations in the variable $x$, and this also requires a uniform bound of the local $L^{p}$-norms of $f$.

Theorem 1.2. Let $F \in C\left(\mathbb{R}^{n} \times \mathbb{R} \times \mathbb{R}^{n} \times \mathcal{S}^{n}\right)$ satisfy the structure condition (SC). Suppose also that for all $R>0$ there exist a constant $K_{R}>0$ and a function $\omega_{R}: \mathbb{R}_{+} \rightarrow \mathbb{R}_{+}$such that $\lim _{t \rightarrow 0^{+}} \omega_{R}(t)=0$ and

$$
|F(y, u, \xi, X)-F(x, u, \xi, X)| \leq K_{R}\|X\||y-x|+\omega_{R}((1+|\xi|)|y-x|)
$$

as $x, y \in \mathbb{R}^{n}, u \in(-R, R)$ and $(\xi, X) \in \mathbb{R}^{n} \times \mathcal{S}^{n}$. If $p>p_{0}, f \in C\left(\mathbb{R}^{n}\right)$, and

$$
\|f\|_{M^{p}}:=\sup _{x \in \mathbb{R}^{n}}\|f\|_{L^{p}\left(B_{1}(x)\right)}<+\infty
$$

then (1.1) has a unique C-viscosity solution.

As it can be seen in Section 4, the structure condition (SC) is sufficient by itself for the existence. The uniqueness, as shown in Section 5, relies on a result of Da Lio and Sirakov 
[6] which is fundamental in our proof for the comparison between two solutions $u$ and $v$. By virtue of this result, conditions (A2.1) and (A2.2) imply that the difference $w=u-v$ satisfies a maximal equation with a constant first-order coefficient. In particular, $(A 2.1)$ and (A2.2) are, respectively, stronger than the continuity of $F$ in the $x$-variable and than the local summability of $|f|^{p}$. Later on we also refer to these conditions as to the assumption:

$$
(A 2):=(A 2.1) \text { and }(A 2.2) \text {. }
$$

In order to deal with $F$ merely measurable in $x$, we will suppose for every $R>0$ there exists $c_{R}>0$ such that

$$
\begin{aligned}
& D_{\lambda, \Lambda}^{-}(Y-X)-\gamma|\eta-\xi|-c_{R}|v-u| \\
& \quad \leq F(x, v, \eta, Y)-F(x, u, \xi, X) \leq D_{\lambda, \Lambda}^{+}(Y-X)+\gamma|\eta-\xi|+c_{R}|v-u|
\end{aligned}
$$

for $x \in \mathbb{R}^{n}$ and $u, v \in(-R, R), \xi, \eta \in \mathbb{R}^{n}, X, Y \in \mathcal{S}^{n}$. We put

$$
(\overline{\mathrm{SC}}):=(1.13) \text { and }(1.3) \text { and }(1.4) \text {. }
$$

Then, we say that $F$ satisfies $C^{1,1}$-estimates at $x_{0}$ if for all $w_{0} \in C^{0}\left(\partial B_{r_{0}}\left(x_{0}\right)\right)$ there exists a solution $u \in C^{2}\left(B_{r_{0}}\left(x_{0}\right)\right) \cap C^{0}\left(\bar{B}_{r_{0}}\left(x_{0}\right)\right)$ of the Dirichlet problem

$$
F\left(x_{0}, 0,0, D^{2} w\right)=0 \quad \text { in } B_{r_{0}}\left(x_{0}\right), \quad w=w_{0} \quad \text { on } \partial B_{r_{0}}\left(x_{0}\right)
$$

such that

$$
\|u\|_{C^{1,1}\left(B_{r_{0} / 2}\left(x_{0}\right)\right)} \leq C r_{0}^{-2}\|w\|_{L^{\infty}\left(\partial B_{r_{0}}\left(x_{0}\right)\right)}
$$

for some $r_{0}>0$.

Finally, let

$$
\beta_{F}\left(x, x_{0}\right):=\sup _{\substack{X \in S^{n} \\ X \neq 0}} \frac{\left|F(x, 0,0, X)-F\left(x_{0}, 0,0, X\right)\right|}{\|X\|}
$$

In the case $p>n$, by Caffarelli [7], if $\beta_{F}\left(x, x_{0}\right)$ is sufficiently "small" in a sense that will be made precise below, then $u \in W_{\text {loc }}^{2, p}\left(B_{r_{0}}\left(x_{0}\right)\right)$. Such result was generalized by Escauriaza to the range $p>p_{0}$ with $p_{0} \in(n / 2, n)$ introduced above.

As a consequence, the structure conditions $(\overline{\mathrm{SC}})$ can be used "pointwise" to compare $F\left(x, v(x), D v(x), D^{2} v(x)\right)$ with $F\left(x, u(x), D u(x), D^{2} u(x)\right)$ almost everywhere obtaining a maximal equation for the difference $u(x)-v(x)$ to which GMP is applicable.

By virtue of the results of Winter [8] (see also Swiech [9]), the argument can be generalized to the case of $F$ merely measurable in the variable $x$ provided $F$ is convex in the matrix-variable $X$. 
Theorem 1.3. Let $F: \mathbb{R}^{n} \times \mathbb{R} \times \mathbb{R}^{n} \times \mathcal{S}^{n} \rightarrow \mathbb{R}$ be a function satisfying the structure condition $(\overline{\mathbf{S C}})$ almost everywhere $x \in \mathbb{R}^{n}$ such that one of the following assumptions blocks holds true:

(A3) $F$ is continuous and has $C^{1,1}$-estimates for each $x \in \mathbb{R}^{n}$ with some $r_{0}>0$;

$(\mathrm{A} 3)^{\prime} F$ is measurable in $x$ for all $(u, \xi, X) \in \mathbb{R} \times \mathbb{R}^{n} \times \mathcal{S}^{n}$ and convex in $X$.

Let $f \in C\left(\mathbb{R}^{n}\right)$ in the case (A3), $f \in L_{\mathrm{loc}}^{p}\left(\mathbb{R}^{n}\right)$ in the case (A3)' . If

$$
\sup _{r \in\left(0, r_{0}\right)}\left(f_{B_{r}(x)}\left|\beta_{F}(x, y)\right|^{n} d y\right)^{1 / n} \leq \theta
$$

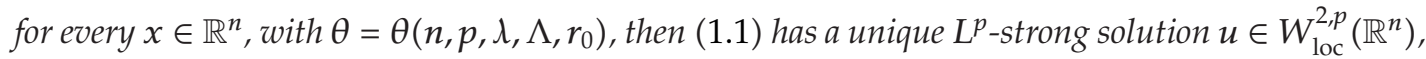
provided that $p>p_{0}$.

By our assumptions, the $L^{p}$-strong solution of Theorem 1.3 will be also the unique $L^{p_{-}}$ viscosity solution.

Theorem 1.3 can be used for instance in the case of Bellman-type equations

$$
\sup _{\alpha}\left\{L_{\alpha} u-f_{\alpha}(x)\right\}=0,
$$

where $L_{\alpha}$ is a semilinear second-order operator

$$
L_{\alpha} u:=a_{\alpha}^{i j}(x) D_{i j} u+b_{\alpha}^{i}(x) D_{i} u+c(x) u+d(x)|u|^{s-1} u
$$

with bounded measurable coefficients such that

$$
\lambda \leq \sup _{|\xi| \leq 1}\left\langle a_{\alpha}^{i j}(x) \xi, \xi\right\rangle \leq \Lambda, \quad\left|b_{\alpha}^{i}(x) b_{\alpha}^{i}(x)\right|^{1 / 2} \leq \gamma, \quad c(x) \leq 0, \quad d(x) \leq-\delta<0
$$

for almost everywhere $x \in \mathbb{R}^{n}$ and every $\alpha$, provided the $a_{\alpha}^{i j}$ are uniformly continuous in $\mathbb{R}^{n}$ with continuity modulus independent of $\alpha$ and $\inf _{\alpha} f_{\alpha} \in L_{\mathrm{loc}}^{p}\left(\mathbb{R}^{n}\right)$.

Some cases of Isaacs-type equations can be treated with Theorem 1.3, as for instance (see [10]), the minimum

$$
\min \left\{\inf _{\alpha}\left(L_{\alpha} u-f_{\alpha}(x)\right), \sup _{\beta}\left(L_{\beta} u-f_{\beta}(x)\right)\right\}=0
$$

between concave and convex operators, which are realized as infimum and supremum, respectively, of two families of semilinear operators, indexed by $\alpha$ and $\beta$, with the above conditions.

Next, consider a regular domain $\Omega \subsetneq \mathbb{R}^{n}$. If $\Omega$ is bounded, in the case of a continuous $F$, condition (SC) is sufficient in order that the Dirichlet problem with continuous boundary conditions has a $C$-viscosity solution by [11, Theorem 1.1]. If $F$ is merely measurable, we will 
use the stronger condition (SC) $)^{\prime}$ of Section 4 needed for the existence of $L^{n}$-viscosity solution in [11, Theorem 4.1].

The technique of the existence part of Theorem 1.3 allows to generalize such results to any regular domain, even unbounded, of $\mathbb{R}^{n}$. For other results in unbounded domains we refer to [12], where the case $s=1$ is considered limiting the growth of $f$.

Theorem 1.4. Let $\Omega \subsetneq \mathbb{R}^{n}$ be a domain satisfying a uniform exterior cone condition, and let $F(x, t, \xi, X)$ be measurable in $x \in \mathbb{R}^{n}$ for all $(t, \xi, X) \in \mathbb{R} \times \mathbb{R}^{n} \times \mathcal{S}^{n}$ such that the structure condition $(\mathrm{SC})^{\prime}$ holds almost everywhere $x \in \Omega$. Then, for $p>p_{0}$ the Dirichlet problem

$$
F\left(x, u, D u, D^{2} u\right)=f(x) \quad \text { in } \Omega, \quad u(x)=\psi(x) \quad \text { on } \partial \Omega
$$

has an $L^{p}$-viscosity solution $u \in C(\bar{\Omega})$ for every $f \in L_{\mathrm{loc}}^{p}\left(\mathbb{R}^{n}\right)$ and every $\psi \in C(\partial \Omega)$.

Remark 1.5. The solution $u \in C(\bar{\Omega})$ of Theorem 1.4 is unique in the cases of Theorems 1.1, 1.2 and 1.3, where the structural and the additional conditions are to be intended correspondingly to hold for $x \in \Omega$ instead of $x \in \mathbb{R}^{n}$.

Finally, a monotonicity argument can instead be used when $f$ is bounded from below to obtain boundary blow-up $L^{p}$-viscosity solutions.

Theorem 1.6. Let $\Omega \subsetneq \mathbb{R}^{n}$ be a domain satisfying a uniform exterior cone condition, and suppose that at least one of the assumption blocks of Theorems 1.1, 1.2 and 1.3 holds true for $x \in \Omega$. Then, for $p>p_{0}$ the Dirichlet problem

$$
F\left(x, u, D u, D^{2} u\right)=f(x) \quad \text { in } \Omega, \quad \lim _{x \rightarrow \partial \Omega} u(x)=+\infty
$$

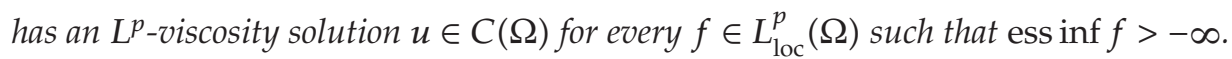

Remark 1.7. Generally the existence results for the BVP of Theorems 1.4 and 1.6 fail to hold if the domain is not sufficiently regular. In fact, assuming $F=\bar{F}(X)$ uniformly elliptic such that

$$
1 \leq \frac{\Lambda}{\lambda}<n-1, \quad s \geq \frac{(n-1) \lambda+\Lambda}{(n-1) \lambda-\Lambda},
$$

Labutin [13] showed that the origin is a removable singularity for the equation

$$
\bar{F}\left(D^{2} u\right)-|u|^{s-1} u=0
$$

that is every $L^{n}$-viscosity solution in the punctured ball $B_{R} \backslash\{0\}$ can be continued to an $L^{n_{-}}$ viscosity solution in $B_{R}$.

The paper is organized as follows. In Section 2 we introduce the notations and recall the main features of viscosity solutions which will be used. Then in Section 3 we prove a locally uniform bound which is the basic tool to construct the solutions in unbounded 
domains. The proof of the existence results will be given in Section 4, while the issue of uniqueness and blow-up is dealt with in Section 5.

\section{Preliminaries}

We will consider functions $F: \Omega \times \mathbb{R} \times \mathbb{R}^{n} \times \mathcal{S}^{n} \rightarrow \mathbb{R}$ where $\Omega$ is a domain (open connected set) of $\mathbb{R}^{n}$ and $\mathcal{S}^{n}$ the space of $n \times n$ real symmetric matrices. The identity matrix will be denoted by $I$ and the trace of $X \in \mathcal{S}^{n}$ with $\operatorname{Tr}(X)$, while $\|X\|$ is one of the equivalent norms of $X$ in $\mathcal{S}^{n}$. For $X, Y \in \mathcal{S}^{n}$ we put $X \leq Y$ if

$$
\langle X \xi, \xi\rangle \leq\langle Y \xi, \xi\rangle \quad \forall \xi \in \mathbb{R}^{n}
$$

We say that $F$ is uniformly elliptic with ellipticity constants $\lambda>0$ and $\Lambda \geq \lambda$ if

$$
p_{\lambda, \Lambda}^{-}(Y-X) \leq F(x, t, \xi, Y)-F(x, t, \xi, X) \leq D_{\lambda, \Lambda}^{+}(Y-X)
$$

for all $(x, t, \xi) \in \Omega \times \mathbb{R} \times \mathbb{R}^{n}$ and $X, Y \in \mathcal{S}^{n}$, where

$$
p_{\lambda, \Lambda}^{+}(Z)=\sup _{\lambda I \leq A \leq \Lambda I} \operatorname{Tr}(A Z), \quad P_{\lambda, \Lambda}^{-}(Z)=\inf _{\lambda I \leq A \leq \Lambda I} \operatorname{Tr}(A Z), \quad Z \in \mathcal{S}^{n}
$$

are, respectively, the maximal and the minimal (Pucci) operators in the class of uniformly elliptic operators with ellipticity constants $\lambda$ and $\Lambda$.

For $u \in C^{2}(\Omega)$ denote by $D u$ and $D^{2} u$ the gradient and the Hessian matrix of $u$. We wish to discuss the solvability of equation

$$
F\left(x, u(x), D u(x), D^{2} u(x)\right)=f(x)
$$

under the assumptions (1.2), (1.3) and (1.4), and we refer to $u \in C^{2}(\Omega)$ satisfying (2.4) for all $x \in \Omega$ as classical solutions of the equation $F=f$ in $\Omega$. If $u \in W_{\text {loc }}^{2, p}(\Omega)$ and the equation is satisfied almost everywhere in $\Omega$, we call it an $L^{p}$-strong solution.

We are interested in the weaker notion of solution in the viscosity sense.

Firstly suppose that $F$ is continuous in $\Omega \times \mathbb{R} \times \mathbb{R}^{n} \times \mathcal{S}^{n}$ and $f$ continuous in $\Omega$. The function $u \in C(\Omega)$ is a $C$-viscosity subsolution, respectively supersolution, of the equation $F\left(x, u, D u, D^{2} u\right)=f$ in $\Omega$ if

$$
F\left(x, u(x), D \varphi(x), D^{2} \varphi(x)\right) \geq f(x)
$$

respectively

$$
F\left(x, u(x), D \varphi(x), D^{2} \varphi(x)\right) \leq f(x)
$$


for each $x \in \Omega$ and $\varphi \in C^{2}\left(B_{r}(x)\right)$ such that $u-\varphi$ has a local maximum, respectively minimum, in $x$. A function $u \in C(\Omega)$ that is both a subsolution and a supersolution in the above sense is a $C$-viscosity solution. $x=0$.

Here and below we denote by $B_{r}(x)$ the ball of radius $r$ centered at $x$, for short $B_{r}$ if

Remark 2.1. If $u \in C^{2}(\Omega)$ is a classical subsolution (supersolution) of $F\left(x, u, D u, D^{2} u\right)=f$, then $u$ is a $C$-viscosity subsolution (supersolution) of $F\left(x, u, D u, D^{2} u\right)=f$. Conversely, if $u \in C^{2}(\Omega)$ is a $C$-viscosity subsolution (supersolution), then $u$ is a classical subsolution (supersolution); see [14, Corollary 2.6].

In the sequel we will also use the fact that, if $u \in C(\Omega)$ is a $C$-viscosity subsolution, respectively supersolution, of $F=f$ and $v \in C(\Omega)$ is a $C$-viscosity subsolution, respectively supersolution, of $F=g$ in $\Omega$, then the function $w=\max (u, v)$, respectively $w=\min (u, v)$, is a $C$-viscosity subsolution, respectively supersolution, of the equation

$$
F\left(x, w, D w, D^{2} w\right)=h
$$

in $\Omega$, where $h=\min (f, g)$, respectively $h=\max (f, g)$.

Lemma 2.2. Let $u, v \in C(\Omega)$ be, respectively $C$-viscosity subsolution and supersolution of the equations $F\left(x, u, D u, D^{2} u\right)=f$ and $F\left(x, v, D v, D^{2} v\right)=g$ in $\Omega$, and assume (1.2)-(1.3). If at least one between $u$ and $v$ is in $C^{2}(\Omega)$, then the difference $w=u-v$ is a $C$-viscosity subsolution of the maximal equation

$$
p_{\lambda, \Lambda}^{+}\left(D^{2} w\right)+\gamma|D w|-\delta w^{s}=f-g
$$

in $\Omega \cap\{w>0\}$.

Proof. Let us suppose, for instance, that $v \in C^{2}(\Omega)$. Let $\varphi$ be a $C^{2}$-function such that $w-\varphi$ has a local maximum in $x \in \Omega \cap\{w>0\}$, then $v+\varphi$ is a test function for $u$, and by structure conditions (1.2)-(1.3) we have

$$
\begin{aligned}
f(x)-g(x) & \leq F\left(x, u(x), D(v+\varphi)(x), D^{2}(v+\varphi)(x)\right)-F\left(x, v(x), D v(x), D^{2} v(x)\right) \\
& \leq D_{\lambda, \Lambda}^{+}\left(D^{2} \varphi(x)\right)+\gamma|D \varphi(x)|-\delta(w(x))^{s}
\end{aligned}
$$

as claimed.

When $F$ is merely measurable in $x$, we assume that the structure condition (SC) holds for almost every $x \in \Omega$. Note that, if $F$ is continuous in $x$, then (1.2) implies the uniform ellipticity.

Let $f \in L_{\text {loc }}^{p}(\Omega)$; then $u \in C(\Omega)$ is called an $L^{p}$-viscosity subsolution, respectively supersolution, of the equation $F\left(x, u, D u, D^{2} u\right)=f$ in $\Omega$ if

$$
\text { ess } \limsup _{x \rightarrow \bar{x}}\left\{F\left(x, u(x), D \varphi(x), D^{2} \varphi(x)\right)-f(x)\right\} \geq 0
$$


respectively

$$
\text { ess } \liminf _{x \rightarrow \bar{x}}\left\{F\left(x, u(x), D \varphi(x), D^{2} \varphi(x)\right)-f(x)\right\} \leq 0,
$$

for each $\bar{x} \in \Omega$ and $\varphi \in W^{2, p}\left(B_{r}(\bar{x})\right)$ such that $u-\varphi$ has a local maximum, respectively minimum, in $\bar{x}$. A function $u \in C(\Omega)$ that is both a subsolution and a supersolution is an $L^{p}$-viscosity solution.

It is important that the generalized maximum principle (GMP) for $L^{p}$-strong solutions of the maximal equation (see (1.10) at the beginning of the Introduction) continues to hold for $L^{p}$-viscosity subsolutions as $p>p_{0}$; see for instance [5, Theorem 3.2].

Note that $L^{p}$-viscosity solutions are $C$-viscosity solutions of $F\left(x, u, D u, D^{2} u\right)=f$ because the space $W_{\text {loc }}^{2, p}(\Omega)$ of test functions for $L^{p}$-viscosity solutions is larger than $C^{2}(\Omega)$.

Conversely, if $p>p_{0}$, assuming that $F$ and $f$ are continuous, then $C$-viscosity solutions are $L^{p}$-viscosity solutions; see [15, Proposition 2.9].

Remark 2.3. Suppose that $p>p_{0}$, as before. If $u \in W_{\text {loc }}^{2, p}(\Omega)$ is an $L^{p}$-strong subsolution (supersolution) of $F\left(x, u, D u, D^{2} u\right)=f$, then $u$ is also an $L^{p}$-viscosity subsolution (supersolution); see [15, Lemma 2.6].

Conversely, if $u \in W_{\text {loc }}^{2, p}(\Omega)$ is an $L^{p}$-viscosity subsolution (supersolution), then $u$ is an $L^{p}$-strong subsolution (supersolution); see [15, Corollary 3.7].

Also, Remark 2.1 for $w=\max (u, v)$, respectively $w=\min (u, v)$, continues to hold for $L^{p}$-viscosity subsolutions, respectively supersolutions, $u, v \in C(\Omega)$.

Finally, assuming (1.2)-(1.3) almost everywhere in $\Omega$, we infer that Lemma 2.2 continues to hold for $L^{p}$-viscosity solutions.

For an extensive treatment of viscosity solutions see [11, 15-17].

In the existence results we need some regularity of the domain $\Omega$ of $\mathbb{R}^{n}$. We say that $\Omega$ satisfies an exterior cone condition if for every point $\bar{x} \in \partial \Omega$ there exists a finite right circular cone $\Sigma_{\bar{x}}$ with vertex $\bar{x}$ such that $\bar{\Sigma}_{\bar{x}} \cap \bar{\Omega}=\{\bar{x}\}$. A uniform exterior cone condition means that all the cones $\Sigma_{\bar{x}}$ are congruent to a fixed cone $\Sigma$. For the use of these conditions see, for instance, [18] about the $L^{p}$ theory and [11] in the viscosity setting.

\section{Uniform Estimates}

In this section, following [2], we introduce Osserman's barrier function

$$
\Phi(x)=C_{R} R^{\mu} \xi^{-\mu}(x), \quad|x|<R,
$$

where $\xi(x)=R^{2}-|x|^{2}$ and $\mu=2 /(s-1)$ (recall that $\left.s>1\right)$.

Lemma 3.1. Suppose for almost everywhere $x \in B_{R}$ that

$$
F(x, v, \xi, X) \leq D_{\lambda, \Lambda}^{+}(X)+\gamma|\xi|-\delta v^{s}
$$

for all $(v, \xi, X) \in \mathbb{R}_{+} \times \mathbb{R}^{n} \times \mathcal{S}^{n}$, where $\delta>0$ and $s>1$. 
If one takes

$$
C_{R}^{s-1}=2 \mu \delta^{-1}(\Lambda(n+2(1+\mu))+\gamma R)
$$

then the function $\Phi \in C^{2}\left(B_{R}\right)$, defined in (3.1), is an $L^{p}$-strong solution of the equation

$$
F\left(x, \Phi, D \Phi, D^{2} \Phi\right) \leq 0 \quad \text { in } B_{R}
$$

Proof. By the assumptions, it is sufficient to show that

$$
p_{\lambda, \Lambda}^{+}\left(D^{2} \Phi\right)+\gamma|D \Phi|-\delta \Phi^{s} \leq 0
$$

Since $\Phi(x)=\varphi(r):=C_{R} R^{\mu}\left(R^{2}-r^{2}\right)^{-\mu}$, where $r=|x|$, then

$$
p_{\lambda, \Lambda}^{+}\left(D^{2} \Phi\right)+\gamma|D \Phi|-\delta \Phi^{s}=\Lambda\left[\varphi^{\prime \prime}+\frac{n-1}{r} \varphi^{\prime}\right]+\gamma \varphi^{\prime}-\delta \varphi^{s},
$$

and the result follows by choosing $C_{R}>0$ as claimed.

In what follows $p_{0} \in(n / 2, n)$ is the exponent such that GMP holds for $p>p_{0}$ (see Sections 1 and 2) and $u^{ \pm}=\max ( \pm u, 0)$.

Lemma 3.2. Let $\Omega$ be a domain of $\mathbb{R}^{n}$ such that $\Omega_{R}:=\Omega \cap B_{R} \neq \emptyset$. Suppose that $F$ satisfies structure conditions (SC) almost everywhere $x \in \Omega_{R}$. If $u \in C\left(\bar{\Omega}_{R}\right)$ is an $L^{p}$-viscosity solution $\left(p>p_{0}\right)$ of the equation

$$
F\left(x, u, D u, D^{2} u\right) \geq f(x)
$$

with $f \in L^{p}\left(\Omega_{R}\right)$, then for each $r \in(0, R)$ one has

$$
\sup _{\Omega_{r}} u \leq u_{\partial \Omega}^{+}+\frac{C_{0}(1+R)^{\mu / 2} R^{\mu}}{\left(R^{2}-r^{2}\right)^{\mu}}+C\left\|f^{-}\right\|_{L^{p}\left(\Omega_{R}\right)}
$$

where $\mu=2 /(s-1)$, and $C_{0}=C_{0}(n, \Lambda, \gamma, s, \delta)$ and $C=C(n, p, \Lambda, \Lambda, \gamma R)$ are positive constants.

Here,

$$
u_{\partial \Omega}^{+}= \begin{cases}\sup _{B_{R} \cap \partial \Omega} u^{+} & \text {if } B_{R} \cap \partial \Omega \neq \emptyset \\ 0 & \text { if } B_{R} \cap \Omega\end{cases}
$$

Proof. By (SC) we have

$$
\begin{aligned}
F(x, v, \xi, X) & =F(x, v, \xi, X)-F(x, v, 0,0)+F(x, v, 0,0) \\
& \leq D_{\lambda, \Lambda}^{+}(X)+\gamma|\xi|-\delta v^{s}
\end{aligned}
$$


for all $(v, \xi, X) \in \mathbb{R}_{+} \times \mathbb{R}^{n} \times \mathcal{S}^{n}$. Thus, from Lemma 3.1 we deduce that $\Phi$ is an $L^{p}$-strong supersolution of the equation

$$
F\left(x, \Phi, D \Phi, D^{2} \Phi\right)=0 \quad \text { in } \Omega_{R}
$$

On the other hand $u$ is an $L^{p}$-viscosity subsolution of the equation

$$
F\left(x, u, D u, D^{2} u\right)=f(x) \text { in } \Omega_{R}
$$

Hence, by Lemma 2.2 (see Remark 2.3) the function $w=u-\Phi$ is an $L^{p}$-viscosity solution of the equation

$$
p_{\lambda, \Lambda}^{+}\left(D^{2} w\right)+\gamma|D w| \geq p_{\lambda, \Lambda}^{+}\left(D^{2} w\right)+\gamma|D w|-\delta w^{s} \geq f(x)
$$

in $A=\Omega_{R} \cap\{u>\Phi\}$.

Let $r \in(0, R)$ be such that $u^{+}(x) \leq \Phi(x)$ as $x \in \Omega$ and $r \leq|x|<R$; then $A \subset \Omega_{r}$ and $w \leq 0$ on $\Omega \cap \partial B_{r}$. Therefore, applying GMP (1.10), we get

$$
\sup _{A} w \leq \sup _{B_{r} \cap \partial \Omega} w+C\left\|f^{-}\right\|_{L^{p}\left(\Omega_{R}\right)}
$$

from which

$$
u(x) \leq \frac{C_{R} R^{\mu}}{\left(R^{2}-|x|^{2}\right)^{\mu}}+u_{\partial \Omega}^{+}+C\left\|f^{-}\right\|_{L^{p}\left(\Omega_{R}\right)}
$$

for all $x \in \Omega_{R}$.

Proposition 3.3. Let $\Omega_{R}, F$, and $f$ be as in Lemma 3.2. If $u \in C\left(\bar{\Omega}_{R}\right)$ is an $L^{p_{\text {-viscosity }} \text { solution }}$ $\left(p>p_{0}\right)$ of the equation

$$
F\left(x, u, D u, D^{2} u\right)=f(x)
$$

then for each $r \in(0, R)$ one has

$$
\sup _{\Omega_{r}}|u| \leq|u|_{\partial \Omega}+\frac{C_{0}(1+R)^{\mu / 2} R^{\mu}}{\left(R^{2}-r^{2}\right)^{\mu}}+C\|f\|_{L^{p}\left(\Omega_{R}\right)}
$$

with $C_{0}, C$ and $|u|_{\partial \Omega}=\max \left(u_{\partial \Omega}^{+}, u_{\partial \Omega}^{-}\right)$as defined in Lemma 3.2. 
Proof. From Lemma 3.2 we already know that

$$
\sup _{\Omega_{r}} u^{+} \leq u_{\partial \Omega}^{+}+\frac{C_{0}(1+R)^{\mu / 2} R^{\mu}}{\left(R^{2}-r^{2}\right)^{\mu}}+C\left\|f^{-}\right\|_{L^{p}\left(\Omega_{R}\right)} .
$$

The assertion will be proved showing the same inequality for $u^{-}$.

To this end firstly observe that the function $v=-u$ satisfies the equation

$$
G\left(x, v, D v, D^{2} v\right)=-f(x) \text { in } \Omega_{R}
$$

where

$$
G(x, v, \xi, X)=-F(x,-v,-\xi,-X),
$$

which turns out to satisfy (SC).

Therefore, $u^{-}=\max (v, 0)$ is an $L^{p}$-viscosity solution of the equation

$$
G\left(x, u^{-}, D u^{-}, D^{2} u^{-}\right) \geq-f(x) .
$$
equation

On the other hand, by virtue of Lemma 3.1, $\Phi$ is an $L^{p}$-strong supersolution of the

$$
p_{\lambda, \Lambda}^{+}\left(D^{2} \Phi\right)+\gamma|D \Phi|-\delta \Phi^{S}=0
$$

Hence, we finish the proof arguing as in Lemma 3.2 to obtain the estimate

$$
u^{-}(x) \leq u_{\partial \Omega}^{-}+\frac{C_{R} R^{\mu}}{\left(R^{2}-|x|^{2}\right)^{\mu}}+C\left\|f^{+}\right\|_{L^{p}\left(\Omega_{R}\right)}
$$

for all $x \in \Omega_{R}$.

\section{Proof of the Existence Results}

In this section, using the structure condition (SC), or the slightly stronger variant (SC)' defined below, we construct an $L^{p}$-viscosity solution of the equation

$$
F\left(x, u, D u, D^{2} u\right)=f(x)
$$

in $\mathbb{R}^{n}$ under the assumption that $f \in L_{\text {loc }}^{p}\left(\mathbb{R}^{n}\right)$ with $p>p_{0}$, where $p_{0} \in(n / 2, n)$ is the exponent above which GMP holds true; see Sections 1 and 2. 


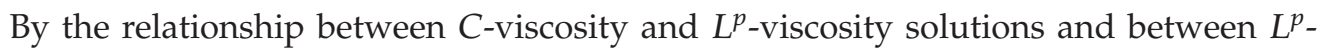
viscosity and $L^{p}$-strong solutions (see Section 2 ) the existence part of each one of Theorems 1.1, 1.2, and 1.3 will follow at once from Proposition 4.1 in rather general assumptions.

We will suppose that for all $R>0$ there exists a function $\omega_{R}: \mathbb{R}_{+} \rightarrow \mathbb{R}_{+}$such that $\omega_{R}(t) \rightarrow 0$ as $t \rightarrow 0^{+}$and

$$
|F(x, v, \xi, X)-F(x, u, \xi, X)| \leq \omega_{R}(|v-u|)
$$

almost everywhere in $x$ for $|u|+|v|+|\xi|+\|X\| \leq R$, observing that it is satisfied by default if we assume that $F$ is a continuous function of $(x, u, \xi, X)$. Then, we put

$$
(\mathbf{S C})^{\prime}:=(\mathbf{S C}) \text { and }(4.2)
$$

It is worth to recall that condition (SC) ${ }^{\prime}$ is equivalent to (SC) in the case that $F$ is continuous.

Proposition 4.1. Let $F: \mathbb{R}^{n} \times \mathbb{R} \times \mathbb{R}^{n} \times \mathcal{S}^{n} \rightarrow \mathbb{R}$ be measurable in $x$ and satisfy the structure condition (SC)' almost everywhere $x \in \mathbb{R}^{n}$ for all $(u, \xi, X) \in \mathbb{R} \times \mathbb{R}^{n} \times \mathcal{S}^{n}$. If $f \in L_{\mathrm{loc}}^{p}\left(\mathbb{R}^{n}\right)$, then equation

$$
F\left(x, u, D u, D^{2} u\right)=f(x)
$$

has an $L^{p}$-viscosity solution in $\mathbb{R}^{n}$ for any $p>p_{0}$.

Proof. Consider a smooth approximation $f_{k} \in C^{\infty}\left(\mathbb{R}^{n}\right)$ of $f$ such that

$$
\lim _{k \rightarrow \infty}\left\|f_{k}-f\right\|_{L^{p}(\Omega)}=0
$$

for every bounded domain $\Omega$ in $\mathbb{R}^{n}$.

By [11, Theorem 4.1, Remark 4.8] we can solve in the $L^{p}$-viscosity sense any Dirichlet problem for the equation $F=f_{k}$ in the ball $B_{2^{k}}$ with continuous boundary condition.

Choose a solution $u_{k}$ for each $k \in \mathbb{N}$. Using Proposition 3.3, for $h>k$ we have

$$
\sup _{B_{2^{k}}}\left|u_{h}\right| \leq C_{0}+C\|f\|_{L^{p}\left(B_{2^{k+1}}\right)}
$$

where $C_{0}=C_{0}(n, \Lambda, \gamma, s, \delta)$ and $C=C\left(n, p, \lambda, \Lambda, \gamma 2^{k+1}\right)$ are positive constants as defined in Lemma 3.2.

By the structure condition (SC)' we have

$$
\begin{aligned}
& F(x, v, \xi, X) \leq D_{\lambda, \Lambda}^{+}(X)+\gamma|\xi|+F(x,-R, 0,0) \leq D_{\lambda, \Lambda}^{+}(X)+\gamma|\xi|+\omega_{R}(R), \\
& F(x, v, \xi, X) \geq D_{\lambda, \Lambda}^{-}(X)-\gamma|\xi|+F(x, R, 0,0) \geq D_{\lambda, \Lambda}^{-}(X)-\gamma|\xi|-\omega_{R}(R),
\end{aligned}
$$

almost everywhere $x \in \mathbb{R}^{n}$ for $|v| \leq R$. 
Therefore for $h>k$ we have

$$
p_{\lambda, \Lambda}^{-}\left(D^{2} u_{h}\right)-\gamma\left|D u_{h}\right|-\omega_{R}(R) \leq f \leq p_{\lambda, \Lambda}^{+}\left(D^{2} u_{h}\right)+\gamma\left|D u_{h}\right|+\omega_{R}(R)
$$

in $B_{2^{k}}$ with $R=C_{0}+C\|f\|_{L^{p}\left(B_{2^{k+1}}\right)}$.

By $C^{\alpha}$-estimates (see [14, Proposition 4.10] and [19, Theorem 2]) we deduce that

$$
\left\|u_{h}\right\|_{C^{\alpha}\left(B_{2^{k}}\right)} \leq C_{1}\left(1+\|f\|_{L^{p}\left(B_{2^{k+1}}\right)}\right)
$$

for a positive constant $C_{1}$ independent of $h>k$.

By a diagonal process, using Ascoli-Arzelà theorem we extract a subsequence $h_{k} \in \mathbb{N}$ such that $u_{h_{k}} \rightarrow u \in C\left(\mathbb{R}^{n}\right)$ uniformly on every bounded domain.

From the stability results for $L^{p}$-viscosity solutions, see [15, Theorem 3.8], $u$ is a solution of the equation.

Proof of Theorem 1.4. In the case $\Omega \subsetneq \mathbb{R}^{n}$ we proceed along the same lines of the proof of Proposition 4.1 constructing $L^{p}$-viscosity solutions $u_{k} \in C\left(\bar{\Omega}_{2^{k}}\right), k \in \mathbb{N}$, of the approximating Dirichlet problems

$$
F\left(x, u_{k}, D u_{k}, D^{2} u_{k}\right)=f_{k}(x) \quad \text { in } \Omega_{2^{k}}, \quad u_{k}(x)=\psi_{k}(x) \quad \text { on } \partial \Omega_{2^{k}}
$$

for a sequence $f_{k} \in C(\Omega)$ such that $\left\|f_{k}-f\right\|_{L^{p}(A)} \rightarrow 0$ for all bounded subsets $A \subset \Omega$.

Here, according to the notations of Proposition 3.3, $\Omega_{2^{k}}=\Omega \cap B_{2^{k}}$, while $\psi_{k}$ is a continuous extension to $\mathbb{R}^{n}$ of $\left.\psi\right|_{\partial \Omega \cap \bar{B}_{2 k}}$; see for instance [20, Section 1.2].

Let $R=2^{k}$. Since $\Omega_{R}$ satisfies in turn a uniform exterior cone property, the existence of such $u_{k}$ follows from the assumptions on $F$ and the already mentioned [11, Theorem 4.1].

Furthermore, by Proposition 3.3, for $h>k$ we get

$$
\sup _{\Omega_{R}}\left|u_{h}\right| \leq \max _{\partial \Omega \cap \bar{B}_{R}}|\psi|+C_{0}+C\|f\|_{L^{p}\left(\Omega_{2 R}\right)} .
$$

The argument of the proof of Lemma 3.2 leads to inequality and therefore $u_{h}$ are equibounded in $\Omega_{R}$.

As a consequence, by $C^{\alpha}$-estimates they are equi-Hölder continuous in every subset

$$
\left\{x \in \Omega_{R} \mid \operatorname{dist}(x, \partial \Omega)>\varepsilon\right\}
$$

with $\varepsilon>0$. By [19, Theorem 2]

$$
\underset{\Omega_{R} \cap B_{\rho}(x)}{\operatorname{OSC}} u_{h} \leq C_{0}\left(\rho^{\alpha_{k}}+\underset{\partial \Omega_{R} \cap B_{\sqrt{\rho}}(x)}{\operatorname{OSCC}} \psi h\right)
$$

for every $x \in \partial \Omega$ and $\rho \leq \rho_{k}$, and therefore $u_{h}$ are also equicontinuous in $\bar{\Omega}_{R}$. 
International Journal of Differential Equations

Thus, using a diagonal procedure as in the proof of Proposition 4.1 we find an $L^{p}$ viscosity solution $u \in C(\bar{\Omega})$ of the Dirichlet problem under consideration.

\section{Uniqueness and Blow-Up}

In this section we begin noticing that from Section 3 we get at once the following maximum principle.

Proposition 5.1. Let $\delta>0, s>1$, and let $\Omega$ be a domain of $\mathbb{R}^{n}$.

Suppose for almost everywhere $x \in \Omega$ that

$$
F(x, u, \xi, X) \leq D_{\lambda, \Lambda}^{+}(X)+\gamma|\xi|-\delta|u|^{s-1} u
$$

for all $(u, \xi, X) \in \mathbb{R} \times \mathbb{R}^{n} \times \mathcal{S}^{n}$ and $u \in C(\bar{\Omega})$ is an $L^{p}$-viscosity solution $\left(p>p_{0}\right)$ of the equation

$$
F\left(x, u, D u, D^{2} u\right) \geq 0 \text { in } \Omega .
$$

(M1) If $\Omega=\mathbb{R}^{n}$, then $u \leq 0$ in $\mathbb{R}^{n}$.

(M2) If $\Omega \subsetneq \mathbb{R}^{n}$ and $u \leq 0$ on $\partial \Omega$, then $u \leq 0$ in $\Omega$.

Analogously, suppose for almost everywhere $x \in \Omega$ that

$$
F(x, v, \xi, X) \geq p_{\lambda, \Lambda}^{-}(X)-\gamma|\xi|-\delta|v|^{s-1} v
$$

for all $(v, \xi, X) \in \mathbb{R} \times \mathbb{R}^{n} \times \mathcal{S}^{n}$ and $v \in C(\bar{\Omega})$ is an $L^{p}$-viscosity solution $\left(p>p_{0}\right)$ of the equation

$$
F\left(x, v, D v, D^{2} v\right) \leq 0 \quad \text { in } \Omega
$$

(m1) If $\Omega=\mathbb{R}^{n}$, then $v \geq 0$ in $\mathbb{R}^{n}$.

(m2) If $\Omega \subsetneq \mathbb{R}^{n}$ and $v \geq 0$ on $\partial \Omega$, then $v \geq 0$ in $\Omega$.

Proof. Let $x \in \mathbb{R}^{n}$ and $r=|x|$. Firstly consider the cases (M1) and (M2). Since

$$
F(x, u, \xi, X)=p_{\lambda, \Lambda}^{+}(X)+\gamma|\xi|-\delta|u|^{s-1} u
$$

satisfies (SC), we can apply Lemma 3.2. Letting $R \rightarrow+\infty$ in (3.8) with $f=0$, we get $u(x) \leq 0$ as asserted.

The other cases (m1) and (m2) can be treated by means of (M1) and (M2) considering the function $u(x)=-v(x)$ and the operator

$$
G(x, u, \xi, X)=-F(x,-u,-\xi,-X)
$$

are as in the proof of Proposition 3.3. 
The above implies that, assuming (SC)', the function $u=0$ is the unique viscosity solution of the problem $F=0$ in $\mathbb{R}^{n}$.

Concerning the uniqueness for the inhomogeneous equation $F=f$ in $\mathbb{R}^{n}$, since solutions are considered in the viscosity sense, we need additional assumptions in order to use "pointwise" the structure conditions and thus to use the above maximum principle.

Also, we cannot in general employ the usual comparison arguments for Dirichlet problems (see for instance [16, Section 3]) not having in principle boundary conditions or bounds at infinity.

Proof of Theorem 1.1 (uniqueness). Let $u$ and $v$ be solutions of the equation $F=f$. Set $\Omega=\{x \in$ $\left.\mathbb{R}^{n} \mid u>v\right\}$. We claim that $\Omega=\emptyset$, so that $u \leq v$ in $\mathbb{R}^{n}$.

Suppose on the contrary that $\Omega \neq \emptyset$. Since $F$ is continuous, arguing as in [21] and observing that $u, v$ are in $C_{\mathrm{loc}}^{1, \alpha}$, we can use the structure condition (SC) to have

$$
p_{\lambda, \Lambda}^{+}\left(D^{2} w\right)+\gamma|D w|-\delta w^{s} \geq 0
$$

in $\Omega$. Using the maximum principle of Proposition 5.1 (M2), we should have $w=u-v \leq 0$ in $\Omega$, a contradiction which proves our claim.

Interchanging the role of $u$ and $v$, we also get $v \leq u$ in $\mathbb{R}^{n}$, and we are done.

Proof of Theorem 1.2 (uniqueness). Here we observe that, if $f \in L_{\mathrm{loc}}^{p}\left(\mathbb{R}^{n}\right)$ and $\|f\|_{M^{p}}<+\infty$, then Proposition 3.3 implies that $u$ is bounded. In fact, if $x_{0} \in \mathbb{R}^{n}$ and we consider balls centered at $x_{0}$, choosing $r \rightarrow 0^{+}$and $R=1$ in (3.17), we get

$$
\left|u\left(x_{0}\right)\right| \leq 2^{\mu / 2} C_{0}+C(n, p, \lambda, \Lambda, \gamma)\|f\|_{M^{p}}
$$

which is finite and independent of $x_{0}$, by $(A 2.2)$.

Thus, if $u$ and $v$ are solutions of the equation $F=f$, by (SC) and (A2.1) we can use of [6, Proposition 2.1] so that the difference $w=u-v$ satisfies a maximal equation

$$
p_{\lambda, \Lambda}^{+}\left(D^{2} w\right)+b|D w|-\delta w^{s} \geq 0
$$

in $\Omega=\left\{x \in \mathbb{R}^{n} \mid u>v\right\}$ for some positive constant $b$ depending on $n, p, \lambda, \Lambda, \gamma$, and $s$.

Therefore, we can conclude as in the proof of Theorem 1.1 (uniqueness).

Proof of Theorem 1.3 (uniqueness). In this case we observe that both the assumptions $(\overline{\mathbf{S C}})$ and (A3) or (A3)' of the Theorem imply that $L^{p}$-viscosity solutions $u, v$ of $F=f$ are in $W_{\text {loc }}^{2, p}\left(\mathbb{R}^{n}\right)$ (see [14, Theorem 7.1], [3, Theorem 1], and [8, Theorem 4.2]). Hence $u, v$ are $L^{p}$-strong solutions (see Remark 2.3), and we can use the structure condition (SC) to get the maximal equation (5.7) for the difference $w=u-v$ in $\Omega=\left\{x \in \mathbb{R}^{n} \mid u>v\right\}$, from which we conclude again using the maximum principle of Proposition 5.1 as in the proof Theorems 1.1 and 1.2 (uniqueness).

We insist on observing that the uniqueness of the solution of the Dirichlet problem depends on the fact that the difference between two solutions is a solution of a homogeneous maximal equation, and then we can invoke the maximum principle of Proposition 5.1. The same method will be used to prove by monotonicity the existence of blow-up solutions. 
Proof of Theorem 1.6. Following [2] we consider a nondecreasing sequence of $f_{k} \in C(\bar{\Omega})$ such that

$$
\lim _{k \rightarrow \infty}\left\|f_{k}-f\right\|_{L^{p}(K)}
$$

for all compact set $K$ of $\bar{\Omega}$. Then by Theorem 1.4 we solve the problem

$$
F\left(x, u_{k}, D u_{k}, D^{2} u_{k}\right)=f_{k}(x) \quad \text { in } \Omega, \quad u_{k}(x)=k \quad \text { on } \partial \Omega
$$

As in the proof of Proposition 4.1, by a diagonal process, using (SC), respectively (SC)', we find an $L^{p}$-viscosity solution $u \in C(\Omega)$ of the equation

$$
F\left(x, u, D u, D^{2} u\right)=f(x) \text { in } \Omega .
$$

To compare $u_{k}$ and $u_{k+1}$, we use (SC) with the additional assumptions (A1) or $(A 2)$, respectively $(\overline{\mathrm{SC}})$ with $(\mathrm{A} 3)$ or $(\mathrm{A} 3)^{\prime}$, to get a maximal equation for $w=u_{k}-u_{k+1}$. Since $f_{k}$ is nondecreasing, then $w$ satisfies the boundary value problem

$$
p_{\lambda, \Lambda}^{+}\left(D^{2} w\right)+b|D w| \geq 0 \quad \text { in } \Omega \cap\left\{u_{k}>u_{k+1}\right\}, \quad w \leq 0 \quad \text { on } \partial\left(\Omega \cap\left\{u_{k}>u_{k+1}\right\}\right)
$$

Hence, using the maximum principle of Proposition 5.1 we get $w \leq 0$, that is $u_{k} \leq u_{k+1}$.

Therefore $u_{k}$ is nondecreasing, and for $\bar{x} \in \partial \Omega$ we have

$$
\lim _{x \rightarrow \bar{x}} u(x) \geq \lim _{x \rightarrow \bar{x}} u_{k}(x)=k
$$

for all $k \in \mathbb{N}$; whence the assertion follows.

\section{References}

[1] H. Brezis, "Semilinear equations in $\mathbb{R}^{n}$ without condition at infinity," Applied Mathematics and Optimization, vol. 12, no. 3, pp. 271-282, 1984.

[2] M. J. Esteban, P. L. Felmer, and A. Quaas, "Superlinear elliptic equation for fully nonlinear operators without growth restrictions for the data," Proceedings of the Edinburgh Mathematical Society, vol. 53, no. 1, pp. 125-141, 2010.

[3] L. Escauriaza, " $W^{2, n}$ a priori estimates for solutions to fully nonlinear equations," Indiana University Mathematics Journal, vol. 42, no. 2, pp. 413-423, 1993.

[4] M. G. Crandall and A. Swiech, "A note on generalized maximum principles for elliptic and parabolic PDE," in Evolution Equations: Proceedings in Honor of J.A. Goldstein's 60th Birthday, vol. 234 of Lecture Notes in Pure and Appl. Math., pp. 121-127, Dekker, New York, NY, USA, 2003.

[5] S. Koike and A. Swiech, "Maximum principle and existence of $L^{p}$ viscosity solutions for fully nonlinear uniformly elliptic equations with measurable and quadratic terms," Nonlinear Differential Equations and Applications, vol. 11, no. 4, pp. 491-509, 2004.

[6] F. Da Lio and B. Sirakov, "Symmetry results for viscosity solutions of fully nonlinear uniformly elliptic equations," Journal of the European Mathematical Society, vol. 9, no. 2, pp. 317-330, 2007.

[7] L. A. Caffarelli, "Interior a priori estimates for solutions of fully nonlinear equations," Annals of Mathematics, vol. 130, no. 1, pp. 189-213, 1989. 
[8] Niki Winter, " $W^{2, p}$ and $W^{1, p}$-estimates at the boundary for solutions of fully nonlinear, uniformly elliptic equations," Zeitschrift für Analysis und ihre Anwendungen, vol. 28, no. 2, pp. 129-164, 2009.

[9] A. Swiech, " $W^{1, p}$-interior estimates for solutions of fully nonlinear, uniformly elliptic equations," Advances in Differential Equations, vol. 2, no. 6, pp. 1005-1027, 1997.

[10] X. Cabré and L. A. Caffarelli, "Interior $C^{2, \alpha}$-regularity theory for a class of nonconvex fully nonlinear elliptic equations," Journal de Mathématiques Pures et Appliquées, vol. 82, no. 5, pp. 573-612, 2003.

[11] M. G. Crandall, M. Kocan, P. L. Lions, and A. Swiech, "Existence results for boundary problems for uniformly elliptic and parabolic fully nonlinear equations," Electronic Journal of Differential Equations, vol. 24, pp. 1-20, 1999.

[12] H. Ishii, "On uniqueness and existence of viscosity solutions of fully nonlinear second-order elliptic PDEs," Communications on Pure and Applied Mathematics, vol. 42, no. 1, pp. 15-45, 1989.

[13] D. A. Labutin, "Removable singularities for fully nonlinear elliptic equations," Archive for Rational Mechanics and Analysis, vol. 155, no. 3, pp. 201-214, 2000.

[14] L. A. Caffarelli and X. Cabré, Fully Nonlinear Elliptic Equations, vol. 43 of Colloquium Publications, American Mathematical Society, Providence, RI, USA, 1995.

[15] L. Caffarelli, M. G. Crandall, M. Kocan, and A. Swiech, “On viscosity solutions of fully nonlinear equations with measurable ingredients," Communications on Pure and Applied Mathematics, vol. 49, no. 4, pp. 365-397, 1996.

[16] M. G. Crandall, H. Ishii, and P. L. Lions, "User's guide to viscosity solutions of second order partial differential equations," American Mathematical Society. Bulletin, vol. 27, no. 1, pp. 1-67, 1992.

[17] S. Koike, A Beginner's Guide to the Theory of Viscosity Solutions, vol. 13 of MSJ Memoirs, Mathematical Society of Japan, Tokyo, Japan, 2004.

[18] D. Gilbarg and N. S. Trudinger, Elliptic Partial Differential Equations of Second Order, vol. 224, Springer, Berlin, Germany, 1983.

[19] B. Sirakov, "Solvability of uniformly elliptic fully nonlinear PDE," Archive for Rational Mechanics and Analysis, vol. 195, no. 2, pp. 579-607, 2010.

[20] L. C. Evans and R. F. Gariepy, Measure theory and fine properties of functions, CRC Press, Boca Raton, Fla, USA, 1992.

[21] I. Capuzzo Dolcetta and A. Vitolo, "Glaeser's type gradient estimates for non-negative solutions of fully nonlinear elliptic equations," Discrete and Continuous Dynamical Systems A, vol. 28, no. 2, pp. 539-557, 2010. 


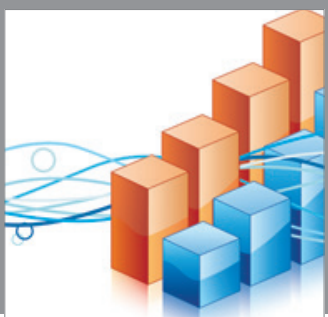

Advances in

Operations Research

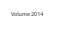

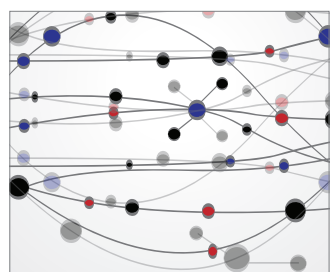

\section{The Scientific} World Journal
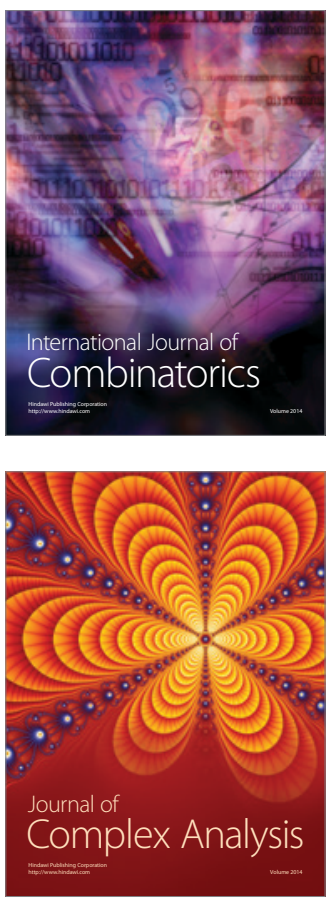

International Journal of

Mathematics and

Mathematical

Sciences
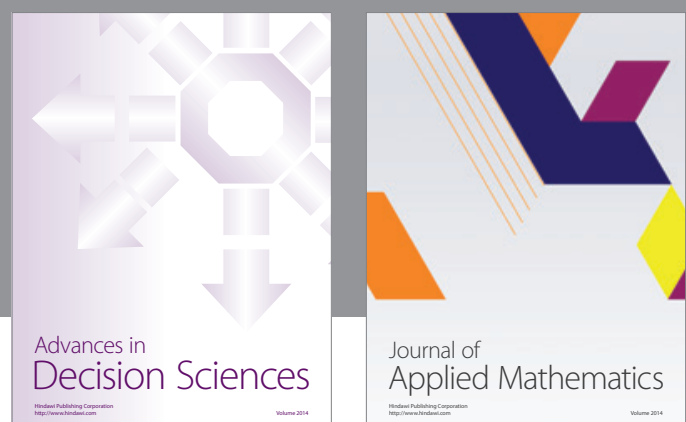

Journal of

Applied Mathematics
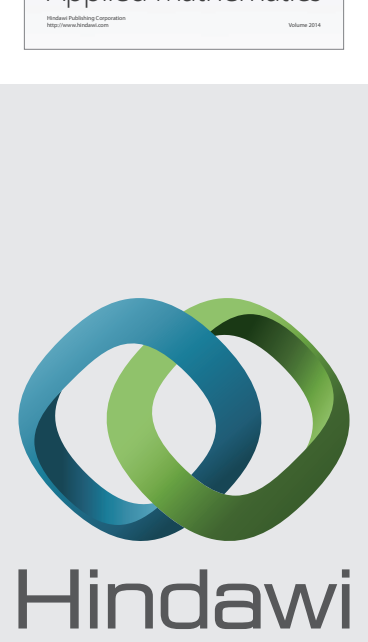

Submit your manuscripts at http://www.hindawi.com
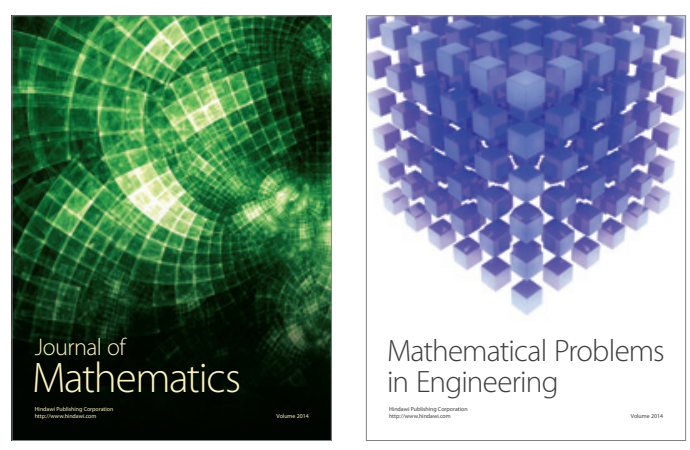

Mathematical Problems in Engineering
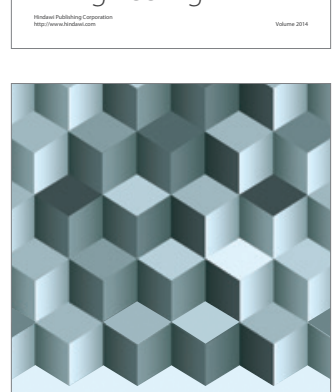

Journal of

Function Spaces
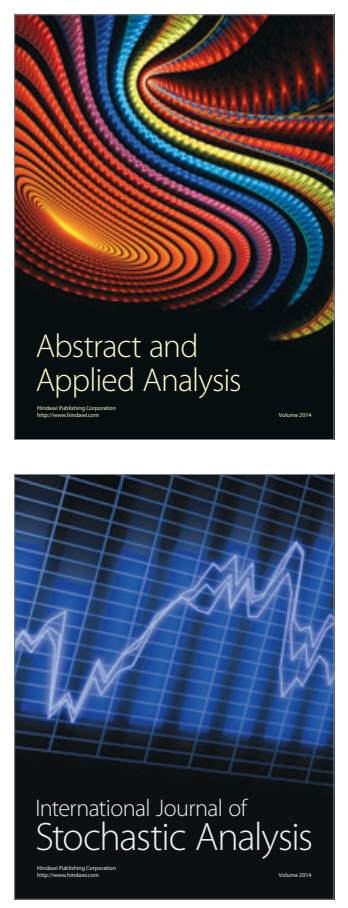

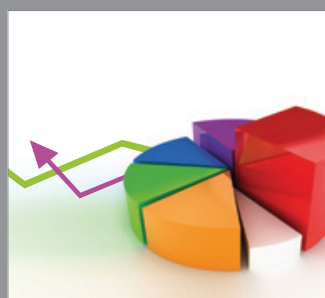

ournal of

Probability and Statistics

Promensencen
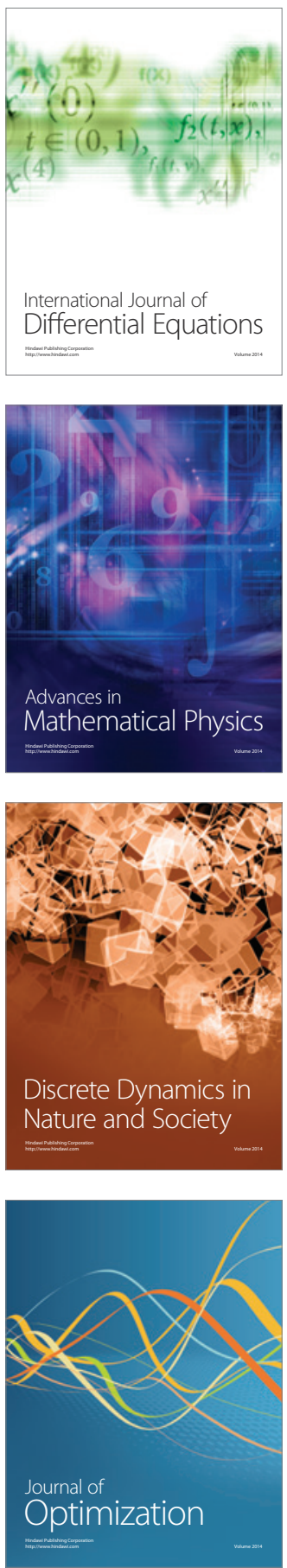